\title{
Solvent-assisted monomeric molecular structure of the phosphate diester and the synthesis of menthol-based phosphate diesters
}

\author{
DEBDEEP MANDAL ${ }^{\mathrm{a}}$, VIVEK GUPTA ${ }^{\mathrm{a}}$, BISWAJIT SANTRA ${ }^{\mathrm{a}}$, NICOLAS CHRYSOCHOS $^{\mathrm{b}}$, \\ VIVEK W BHOYARE $^{\mathrm{c}}$, AVIJIT MAITI ${ }^{\mathrm{a}}$, ABHISEK KAR ${ }^{\mathrm{a}}$, SUMAN $^{\mathrm{a}}$, ANKU GUHA ${ }^{\mathrm{a}}$, \\ PALLAVI THAKUR ${ }^{\mathrm{a}}$, RAMAKIRUSHNAN SURIYA NARAYANAN ${ }^{\mathrm{a}}$, NITIN T PATIL ${ }^{\mathrm{c}, *}$, \\ CAROLA SCHULZKE $^{\mathrm{b}, *}$ (D) VADAPALLI CHANDRASEKHAR ${ }^{\mathrm{a}, \mathrm{d}, *}$ and ANUKUL JANA ${ }^{\mathrm{a}, *}$ (D) \\ ${ }^{\mathrm{a}}$ Tata Institute of Fundamental Research Hyderabad, Gopanpally, Hyderabad 500 107, Telangana, India \\ ${ }^{\mathrm{b}}$ Institut für Biochemie, Universität Greifswald, Felix-Hausdorff-Straße 4, 17487 Greifswald, Germany \\ ${ }^{c}$ Department of Chemistry, Indian Institute of Science Education and Research (IISER)-Bhopal, \\ Bhopal 462 066, Madhya Pradesh, India \\ ${ }^{\mathrm{d}}$ Department of Chemistry, Indian Institute of Technology Kanpur, Kanpur 208 016, Uttar Pradesh, India \\ E-mail: npatil@iiserb.ac.in; carola.schulzke@uni-greifswald.de; vc@iitk.ac.in; ajana@tifrh.res.in
}

MS received 5 August 2019; revised 7 November 2019; accepted 7 November 2019; published online 5 December 2019

\begin{abstract}
Phosphate diesters are well known to form intermolecular H-bonded dimeric structures in their solid-state. Recently, we reported 2,6- $(\mathrm{CHPh})_{2}-4-i$ Pr-phenyl substituted phosphate diester exists as H-bonded monomeric molecular structure along with water dimer in the solid-state. Herein we report $2,6-(\mathrm{CHPh})_{2}-4-$ $i$ Pr-phenyl substituted phosphate diester forms a monomeric molecular structure in the solid-state upon cocrystallization with dimethylformamide, DMF $\left(\mathrm{Me}_{2} \mathrm{NCHO}\right)$. The $-\mathrm{CHO}$ group of DMF simultaneously acts as an $\mathrm{H}$-bond acceptor to $\mathrm{P}-\mathrm{OH}$ and an $\mathrm{H}$-bond donor to $\mathrm{P}=\mathrm{O}$ moieties. We also used the alcohols, $\mathrm{ROH}$ $(\mathrm{R}=\mathrm{Me}, \mathrm{Et}, i \mathrm{Pr}$, and $t \mathrm{Bu})$, for crystallisation of 2,6-(CHPh $)_{2}-4-i \mathrm{Pr}-$ phenyl substituted phosphate diester. In these instances, solvent-incorporated dimeric structures are found in the solid-state. We also report the syntheses and molecular structures of anionic phosphate diesters of 2,6- $(\mathrm{CHPh})_{2}-4-i$ Pr-phenyl substituted phosphate diester possessing various counter cations. Moreover, we also report the syntheses and molecular structures of phosphate diesters based on (-)-menthol, (+)-menthol and $(+) /(-)$-menthol. These exist as H-bonded dimers in the solid-state.
\end{abstract}

Keywords. Phosphate monoesters; phosphate diesters; phosphonic acids; phosphinic acids; X-ray diffraction.

\section{Introduction}

In the solid-state, the existence of an entirely free terminal $\mathrm{P}(\mathrm{O})(\mathrm{OH})$ moiety in phosphate monoesters, phosphate diesters, phosphonic acids and phosphinic acids is not known. ${ }^{1-9}$ Such compounds are present as H-bonded structures either through intermolecular association or through interaction with solvent molecules through the donor $(\mathrm{P}-\mathrm{OH})$ and acceptor $(\mathrm{P}=\mathrm{O})$ moieties. ${ }^{10-25}$ These hydrogen bonding interactions in phosphates lead to the formation of various types of aggregates in the solid-state. ${ }^{26-28}$ Structures of these aggregates depend on the solvent of crystallization and the steric bulk of the substituents. ${ }^{6}$ Aggregation behaviour of para-substituted monoarylphosphates have been very well studied and the formation of $\mathrm{H}$-bonded non-porous and porous 3-D framework structures was reported. ${ }^{29-32}$ Recently Murugavel et al., ${ }^{33}$ and $\mathrm{we}^{34}$ have shown that phosphate monoesters, 2,6- $(\mathrm{CHPh})_{2}-4-\mathrm{R}-\mathrm{C}_{6} \mathrm{H}_{2} \mathrm{O}-\mathrm{P}(\mathrm{O})(\mathrm{OH})_{2}$ form solvent assisted dimeric molecular structures in the solidstate. The corresponding anionic phosphate monoesters derived from these bulky phosphate monoesters exhibit structural diversity in supramolecular

\footnotetext{
*For correspondence
} 
organization based on counter cations ${ }^{35}$ as well as modulation of the nuclearity of molecular $\mathrm{Mg}$ (II)phosphates. ${ }^{36} \mathrm{We}$ further disclosed that the phosphate diester, $\left(2,6-(\mathrm{CHPh})_{2}-4-i \mathrm{Pr}-\mathrm{C}_{6} \mathrm{H}_{2} \mathrm{O}\right)_{2} \mathrm{P}(\mathrm{O}) \mathrm{OH}$, encapsulates a water dimer and as a result is present as a monomer in the solid-state. ${ }^{37}$ In order to examine the influence of solvents on the formation of monomeric or dimeric structures of phosphate diester, (2,6$\left.(\mathrm{CHPh})_{2}-4-i \mathrm{Pr}-\mathrm{C}_{6} \mathrm{H}_{2} \mathrm{O}\right)_{2} \mathrm{P}(\mathrm{O}) \mathrm{OH}$, we have carried out further studies which are reported here. The syntheses of anionic phosphate diesters were also considered and their solid-state molecular structures examined. Moreover, we report the syntheses and molecular structures of phosphate diesters based on stereochemically distinct (-)-menthol, (+)-menthol and $(+) /(-)$-menthol.

\section{Experimental}

\subsection{Materials and general procedures}

Reactions involving $n \mathrm{BuLi}$ and $\mathrm{POCl}_{3}$ were carried out under an argon atmosphere using standard Schlenk techniques and all remaining reactions were carried out under an open atmosphere inside a fume hood. THF was dried by Innovative Technology solvent purification system. Compounds $\mathbf{1} \cdot \mathbf{E t}_{\mathbf{2}} \mathbf{O},{ }^{37} \mathbf{2} \cdot \mathbf{E t}_{\mathbf{3}} \mathbf{N}^{37}$ and $(\mathrm{RO})_{2} \mathrm{POCl}^{38}$ with $\mathrm{R}=(-)$-menthol and/or (+)-menthol were prepared according to literature procedures. NMR spectra were recorded on a BrukerNanoBay 300 $\mathrm{MHz}$ NMR spectrometer. ${ }^{1} \mathrm{H}$ and ${ }^{13} \mathrm{C}\left\{{ }^{1} \mathrm{H}\right\}$ NMR spectra were referenced to the peaks of residual protons of the deuterated solvent $\left({ }^{1} \mathrm{H}\right)$ or the deuterated solvent itself $\left({ }^{13} \mathrm{C}\left\{{ }^{1} \mathrm{H}\right\}\right) .{ }^{31} \mathrm{P}$ NMR spectra were referenced to external $\mathrm{H}_{3} \mathrm{PO}_{4}$. FT-IR spectra were recorded on a Bruker-Alpha spectrometer. Melting points were determined in Stuart melting point apparatus SMP10 and are uncorrected. Electrospray ionization mass spectrometry (ESI-MS) spectra were recorded on a Waters-Q-Tof Premier-HAB213 spectrometer.

\subsection{Synthesis of 1-4}

$2.2 a$ Isolation of 1.DMF Slow evaporation of a dimethylformamide (DMF) $(0.5 \mathrm{~mL})$ solution of $\mathbf{1} \cdot \mathbf{E t}_{\mathbf{2}} \mathbf{O}(0.100 \mathrm{~g})$ at room temperature leads to an almost quantitative formation of 1.DMF.

$2.2 b$ Isolation of $\mathbf{1} \cdot \mathbf{M e O H}$ Slow evaporation of a $\mathrm{CH}_{3} \mathrm{OH}(5 \mathrm{~mL})$ solution of $\mathbf{1} \cdot \mathbf{E t}_{2} \mathbf{O}(0.100 \mathrm{~g})$ at room temperature leads to the almost quantitative formation of $\mathbf{1} \cdot \mathrm{MeOH}$. 2.2c Isolation of 1.EtOH Slow evaporation of an ethanol $(5 \mathrm{~mL})$ solution of $\mathbf{1} \cdot \mathbf{E t}_{\mathbf{2}} \mathbf{O}(0.100 \mathrm{~g})$ at room temperature leads to the almost quantitative formation of 1.EtOH.

\section{2d Isolation of 1-iPrOH}

Method I: Slow evaporation of an isopropanol (5 $\mathrm{mL})$ solution of $\mathbf{1} \cdot \mathbf{E t}_{\mathbf{2}} \mathbf{O}(0.100 \mathrm{~g})$ at room temperature leads to the almost quantitative formation of $\mathbf{1} \cdot \mathbf{i P r O H}$.

Method II: Slow evaporation of an acetone $(1 \mathrm{~mL})$, acetonitrile $(1 \mathrm{~mL})$ and isopropanol $(1 \mathrm{~mL})$ solvent mixture of $\mathbf{1} \cdot \mathbf{E t}_{\mathbf{2}} \mathbf{O}(0.100 \mathrm{~g})$ at room temperature leads to the almost quantitative formation of $\mathbf{1} \cdot \mathbf{i P r O H}$.

2.2e Isolation of 1.tBuOH Slow evaporation of an acetone $(1 \mathrm{~mL})$, acetonitrile $(1 \mathrm{~mL})$ and tertbutanol $(0.5 \mathrm{~mL})$ solvent mixture of $\mathbf{1} \cdot \mathbf{E t}_{\mathbf{2}} \mathbf{O}(0.100 \mathrm{~g})$ at room temperature leads to the almost quantitative formation of $1 \cdot t \mathrm{BuOH}$.

2.2f Synthesis of $\mathbf{2} \cdot \mathbf{i P r}_{2} \mathbf{N E t}$; $\left[\left(\boldsymbol{i P r A r} \mathbf{O}^{\prime}\right)_{2} \boldsymbol{P}(\boldsymbol{O})(\mathrm{O})\right]^{-} \cdot \mathbf{H}-$ $\mathrm{N}(\boldsymbol{E t}) \boldsymbol{i P r}_{2}^{+}$To a solution of $\mathbf{1} \cdot \mathbf{E t}_{\mathbf{2}} \mathbf{O}(0.107 \mathrm{~g}, 0.1 \mathrm{mmol})$ in acetonitrile $(5 \mathrm{~mL})$ was added $\mathrm{EtNiPr}_{2}(0.02 \mathrm{~mL}, 0.1$ $\mathrm{mmol}$ ) and stirred for $5 \mathrm{~h}$. During this period a colorless residue was formed, which was dissolved by addition of DMF ( $2 \mathrm{~mL}$ ) followed by warming. The reaction mixture was filtered and the filtrate kept for crystallization at room temperature affording colorless crystals. Yield: $0.072 \mathrm{~g}$, 64\%. M.p.: $>250{ }^{\circ} \mathrm{C}$. ${ }^{1} \mathrm{H}$ NMR (300 $\mathrm{MHz}, \mathrm{CDCl}_{3}$, $\left.25^{\circ} \mathrm{C}\right): \delta=11.25$ (s, br, $\left.1 \mathrm{H}, \mathrm{N}-H\right), 6.99-6.82(\mathrm{~m}, 40 \mathrm{H}$, $\mathrm{Ar}-H)$, 6.65-6.60 (m, 8H, Ar-H + $\left.\mathrm{CHPh}_{2}\right), 2.94$ (br, 1H,), 2.66 (sept, 2H,CH( $\left.\left(\mathrm{CH}_{3}\right)_{2}\right), 2.48$ (br, 1H,), 2.26 (br, $2 \mathrm{H}, \mathrm{N}\left(\mathrm{CH}\left(\mathrm{CH}_{3}\right)_{2}\right)_{2}, 1.02-0.93\left(\mathrm{~m}, 27 \mathrm{H}, \mathrm{CH}\left(\mathrm{CH}_{3}\right)_{2}+\right.$ $\left.\mathrm{NCH}_{2} \mathrm{CH}_{3}+\mathrm{N}\left(\mathrm{CH}\left(\mathrm{CH}_{3}\right)_{2}\right)_{2}\right)$ ppm. ${ }^{13} \mathrm{C}\left\{{ }^{1} \mathrm{H}\right\}$ NMR (75.431 MHz, $\mathrm{CDCl}_{3}, 25^{\circ} \mathrm{C}$,): $\delta=146.92$ (Ar-Cquart), 146.80 (Ar-Cquart), 144.66 (Ar-Cquart), 143.52 (ArCquart), 136.73 (Ar-Cquart), $129.73(\mathrm{Ar}-\mathrm{CH}), 129.41$ $(\mathrm{Ar}-\mathrm{CH}), 127.91(\mathrm{Ar}-\mathrm{CH}), 127.62(\mathrm{Ar}-\mathrm{CH}), 125.35(\mathrm{Ar}-$ $\mathrm{CH}), \quad 52.46 \quad\left(\mathrm{~N}\left(\mathrm{CH}\left(\mathrm{CH}_{3}\right)_{2}\right)_{2}\right), \quad 49.32 \quad\left(\mathrm{CHPh}_{2}\right), \quad 41.0$ $\left(\mathrm{NCH}_{2} \mathrm{CH}_{3}\right), \quad 33.32\left(\mathrm{CH}\left(\mathrm{CH}_{3}\right)_{2}\right), 23.98\left(\mathrm{CH}\left(\mathrm{CH}_{3}\right)_{2}\right)$, $18.16 \quad\left(\mathrm{~N}\left(\mathrm{CH}\left(\mathrm{CH}_{3}\right)_{2}\right)_{2}\right), \quad 17.16 \quad\left(\mathrm{NCH}_{2} \mathrm{CH}_{3}\right), \quad 11.14$ $\left(\mathrm{NCH}_{2} \mathrm{CH}_{3}\right)$ ppm. ${ }^{31} \mathrm{P}\left\{{ }^{1} \mathrm{H}\right\}$ NMR $\left(121.442 \mathrm{MHz}, \mathrm{CDCl}_{3}\right.$, $\left.25^{\circ} \mathrm{C}\right): \delta=-11.0$ ppm. FT-IR (KBr pellet, $\mathrm{cm}^{-1}$ ): $\bar{v}=3420(\mathrm{w}, \mathrm{br}), 3082(\mathrm{w}), 3057(\mathrm{~m}), 3024(\mathrm{~m}), 2957$ (s), 2870 (w), 2387 (w), 2299 (w), 1943 (w), 1888 (w), 1801 (w), 1599 (m), 1493 (s), 1465 (s), 1445 (s), 1399 (w), 1384 (w), 1362 (w), 1318 (w), 1292 (w), 1244 (s), 1205 (m), 1177 (w), 1159 (m), 1120 (m), 1083 (s), 1031 (m), $920(\mathrm{~m}), 896(\mathrm{~s}), 850(\mathrm{~m}), 829(\mathrm{w}), 796(\mathrm{w}), 763$ (m), $744(\mathrm{w}), 721(\mathrm{~s}), 698(\mathrm{~s}), 666(\mathrm{w}), 646(\mathrm{w}), 628(\mathrm{w})$, $606(\mathrm{~m}), 580(\mathrm{w}), 516(\mathrm{w})$.

\section{$2.2 \mathrm{~g}$ Synthesis of $\mathbf{2} \cdot \mathbf{N H C}^{\mathbf{i P r 2 M e 2} ;} \quad$ [(iPrAr'O $)_{2}$} $\boldsymbol{P}(\boldsymbol{O})(\boldsymbol{O})]^{-} \cdot \boldsymbol{H}-\mathbf{N H C} \boldsymbol{C}^{\boldsymbol{i P r 2 M e 2 +}}$ In a $100 \mathrm{~mL}$ Schlenk 
flask, 1.Et $\mathbf{2} \mathbf{O}(0.072 \mathrm{~g}, 0.07 \mathrm{mmol})$ and $\mathrm{NHC}^{i \operatorname{Pr} 2 \mathrm{Me} 2}$ $(0.013 \mathrm{~g}, 0.07 \mathrm{mmol})$ were mixed. Then dry THF $(20 \mathrm{~mL})$ was added and the resulting reaction mixture was stirred for $5 \mathrm{~h}$ at room temperature. On evaporation of all volatiles followed by washing with diethyl ether $(2 \times 5 \mathrm{~mL})$, a colorless solid was obtained as desired product. Single crystals suitable for X-ray diffraction studies were grown by slow evaporation of acetonitrile solution of $\mathbf{2} \cdot \mathbf{N H C} \mathbf{C}^{i \mathbf{P r 2 M e} 2}$ at room temperature. Yield: 0.048 g, 59\%. M.p.: $>250{ }^{\circ} \mathrm{C}$. ${ }^{1} \mathrm{H}$ $\operatorname{NMR}\left(25^{\circ} \mathrm{C}, 300 \mathrm{MHz}, \mathrm{CDCl}_{3}\right): \delta=10.54(\mathrm{~s}, 1 \mathrm{H}, \mathrm{N}-$ $\mathrm{CH}-\mathrm{N}), 7.06-6.71\left(\mathrm{~m}, 43 \mathrm{H}, \mathrm{Ar}-\mathrm{H}+\mathrm{CHPh}_{2}\right), 6.61(\mathrm{~s}$, $4 \mathrm{H}, \mathrm{Ar}-\mathrm{H}), 5.91$ (s, 1H, CHPh 2$), 4.30$ (sept, 2H, (N$\left.\mathrm{CH}\left(\mathrm{CH}_{3}\right)_{2}\right), 2.65$ (sept, $\left.2 \mathrm{H}, \mathrm{CH}\left(\mathrm{CH}_{3}\right)_{2}\right), 2.16$ (s, 6H, $\left.\mathrm{C}(4,5)-\mathrm{CH}_{3}\right), 1.29\left(\mathrm{~d}, 12 \mathrm{H},\left(\mathrm{N}-\mathrm{CH}\left(\mathrm{CH}_{3}\right)_{2}\right), 1.01\right.$ (d, $\left.12 \mathrm{H}, \mathrm{CH}\left(\mathrm{CH}_{3}\right)_{2}\right) \mathrm{ppm} .{ }^{13} \mathrm{C}\left\{{ }^{1} \mathrm{H}\right\}$ NMR $\left(25{ }^{\circ} \mathrm{C}, 75.431\right.$ $\left.\mathrm{MHz}, \mathrm{CDCl}_{3}\right): \delta=144.95\left(\mathrm{Ar}-C_{\text {quart }}\right), 142.44(\mathrm{Ar}-$ $\left.C_{\text {quart }}\right), 137.30\left(\mathrm{Ar}-C_{\text {quart }}\right), 137.26(\mathrm{~N}-\mathrm{CH}-\mathrm{N}), 134.89$ $\left(\mathrm{Ar}-C_{\text {quart }}\right), 129.80 \quad\left(\mathrm{Ar}-C_{\text {quart }}\right), 127.38(\mathrm{Ar}-\mathrm{CH})$, $126.83(\mathrm{Ar}-\mathrm{CH}), 125.23[C(4,5), \mathrm{Im}], 125.03(\mathrm{Ar}-$ $\mathrm{CH}), \quad 51.36\left(\mathrm{CHPh}_{2}\right), 49.86\left(\mathrm{~N}-\mathrm{CH}\left(\mathrm{CH}_{3}\right)_{2}\right), \quad 33.36$ $\left(\mathrm{CH}\left(\mathrm{CH}_{3}\right)_{2}\right), 24.08\left(\mathrm{~N}-\mathrm{CH}\left(\mathrm{CH}_{3}\right)_{2}\right), 22.36\left(\mathrm{CH}\left(\mathrm{CH}_{3}\right)_{2}\right)$, $8.76\left[\mathrm{C}(4,5)-\mathrm{CH}_{3}\right]$ ppm. ${ }^{31} \mathrm{P}\left\{{ }^{1} \mathrm{H}\right\} \quad \mathrm{NMR}\left(25^{\circ} \mathrm{C}\right.$, $\left.121.442 \mathrm{MHz}, \mathrm{CDCl}_{3}\right): \delta=-9.2 \mathrm{ppm}$. FT-IR $(\mathrm{KBr}$ pellet, $\mathrm{cm}^{-1}$ ): $\bar{v}=3421(\mathrm{~m}, \mathrm{br}), 3117(\mathrm{w}), 3081(\mathrm{w})$, 3057 (m), 3024 (m), 2958 (s), 2865 (m), 2801 (w), 1947 (w), 1884 (w), 1807 (w), 1629 (w), 1599 (m), 1581 (w), 1555 (m), 1493 (s), 1464 (s), 1445 (s), 1393 (w), 1379 (w), 1359 (w), 1319 (w), 1289 (w), 1249 (s), 1202 (m), 1156 (m), 1118 (s), 1096 (s), 1030 (m), $1002(\mathrm{w}), 986(\mathrm{w}), 967(\mathrm{w}), 916(\mathrm{w}), 890(\mathrm{~m}), 878(\mathrm{~s})$, 857 (w), 841 (s), 794 (w), $764(\mathrm{~m}), 719$ (s), 701 (s), $659(\mathrm{~s}), 633(\mathrm{w}), 623(\mathrm{w}), 606(\mathrm{w}), 598(\mathrm{~m}), 588(\mathrm{~m})$, $574(\mathrm{~m}), 546(\mathrm{~m})$. ESI-MS: Calcd $(\mathrm{m} / \mathrm{z})$ for $\left[\mathrm{C}_{11} \mathrm{H}_{21} \mathrm{~N}_{2}^{+}\right]$: 181.1699; found: 181.1613.

\section{$2.2 \mathrm{~h}$ Synthesis of $\mathbf{3} ; \quad\left[\left(\boldsymbol{i P r A r} \mathbf{O}_{2} \boldsymbol{P}(\boldsymbol{O})(\boldsymbol{O})\right]_{2}^{2-}\right.$.} $\mathrm{Zn}(\mathrm{MeOH})_{4}\left(\mathrm{H}_{2} \mathrm{O}\right)_{2}^{2+}$ To a solution of $\mathbf{2} \cdot \mathbf{E t}_{3} \mathbf{N}(0.031 \mathrm{~g}$, $0.028 \mathrm{mmol})$ in methanol $(5 \mathrm{~mL})$ was added a solution of $\mathrm{ZnCl}_{2}(0.004 \mathrm{~g}, 0.028 \mathrm{mmol})$ in methanol $(5 \mathrm{~mL})$ with stirring followed by addition of $\operatorname{DMF}(0.03 \mathrm{~mL})$ at room temperature. The resulting reaction mixture was heated at $70{ }^{\circ} \mathrm{C}$ for $5 \mathrm{~h}$ and during this period a colorless residue was formed which was filtered-off and the filtrate kept for crystallization. Colorless crystals suitable for X-ray diffraction studies were obtained by slow evaporation at room temperature. Yield: $0.012 \mathrm{~g}, 38 \%$. M.p.: $224-228{ }^{\circ} \mathrm{C} .{ }^{1} \mathrm{H}$ NMR $\left(300 \mathrm{MHz}, \mathrm{CDCl}_{3}, 25{ }^{\circ} \mathrm{C}\right)$ : $\delta=6.78-6.98(\mathrm{~m}, 40 \mathrm{H}, \mathrm{Ar}-H), 6.62(\mathrm{~s}, 4 \mathrm{H}, \mathrm{Ar}-H)$, $6.32\left(\mathrm{~s}, \quad 4 \mathrm{H}, \quad \mathrm{CHPh}{ }_{2}\right), \quad 2.57-2.71 \quad(\mathrm{~m}, \quad 16 \mathrm{H}$, $\left.\mathrm{CH}\left(\mathrm{CH}_{3}\right)_{2}+4 \mathrm{CH}_{3} \mathrm{OH}\right), 1.01$ (d, $\left.12 \mathrm{H}, \mathrm{CH}\left(\mathrm{CH}_{3}\right)_{2}\right), 0.83$ (s, 4H, $\left.\mathrm{H}_{2} \mathrm{O}\right) \mathrm{ppm} .{ }^{13} \mathrm{C}\left\{{ }^{1} \mathrm{H}\right\}$ NMR $(75.431 \mathrm{MHz}$, $\left.\mathrm{CDCl}_{3}, \quad 25^{\circ} \mathrm{C}\right): \quad \delta=143.69 \quad\left(\mathrm{Ar}-\mathrm{C}_{\text {quart }}\right), \quad 129.59$
$(\mathrm{Ar}-\mathrm{CH}), 127.85(\mathrm{Ar}-\mathrm{CH}), 127.43(\mathrm{Ar}-\mathrm{CH}), 125.75$ $(\mathrm{Ar}-\mathrm{CH}), 49.76\left(\mathrm{CHPh}_{2}\right), 45.54\left(\mathrm{CH}_{3} \mathrm{OH}\right), 33.37$ $\left(\mathrm{CH}\left(\mathrm{CH}_{3}\right)_{2}\right), 23.93\left(\mathrm{CH}\left(\mathrm{CH}_{3}\right)_{2}\right)$ ppm. ${ }^{31} \mathrm{P}\left\{{ }^{1} \mathrm{H}\right\}$ NMR $\left(121.442 \mathrm{MHz}, \mathrm{CDCl}_{3}, 25^{\circ} \mathrm{C}\right): \delta=-10.6 \mathrm{ppm}$. FT-IR $\left(\mathrm{KBr}\right.$ pellet, $\left.\mathrm{cm}^{-1}\right): \bar{v}=3565(\mathrm{~s}, \mathrm{br}), 3083(\mathrm{w}), 3059$ (m), 3026 (m), 2958 (s), 2869 (w), 1948 (w), $1885(\mathrm{w})$, 1804 (w), 1657 (m), $1599(\mathrm{~m}), 1493$ (s), 1466 (m), 1445 (s), $1383(\mathrm{w}), 1363(\mathrm{w}), 1319(\mathrm{w}), 1291(\mathrm{w}), 1258(\mathrm{~m})$, 1200 (s), 1157 (m), 1117 (s), 1078 (s), 1031 (m), 1003 (w), $966(\mathrm{w}), 925(\mathrm{~s}), 856(\mathrm{~m}), 828(\mathrm{w}), 797(\mathrm{w}), 761$ (m), $746(\mathrm{w}), 722(\mathrm{~s}), 698(\mathrm{~s}), 672(\mathrm{w}), 647(\mathrm{w}), 633(\mathrm{w})$, $621(\mathrm{w}), 605(\mathrm{~m}), 584(\mathrm{w}), 544(\mathrm{w})$.

2.2i Synthesis of $\mathbf{4 a} ;(-) \mathbf{M t}_{\mathbf{2}} \boldsymbol{P}(\boldsymbol{O}) \mathbf{O H}$ (-)-Menthol (5.0 $\mathrm{g}, 32 \mathrm{mmol}$ ) was dissolved in $10 \mathrm{~mL}$ of THF; an equal volume of diethyl ether was added and the mixture was cooled to $-78{ }^{\circ} \mathrm{C}$. The dropwise addition of $n \mathrm{BuLi}(1.6 \mathrm{M}$ in hexane, $20 \mathrm{~mL}, 32 \mathrm{mmol}$ ) resulted in the formation of a colorless suspension. After the mixture was stirred for an additional $10 \mathrm{~min}$ at $-78{ }^{\circ} \mathrm{C}, \mathrm{POCl}_{3}(1.5 \mathrm{~mL}, 16 \mathrm{mmol})$ was slowly added and the solution was warmed to ambient temperature and stirred overnight. All volatiles were removed under vacuum affording a colorless oily compound with $\mathrm{LiCl}$. The resulting reaction mixture was dissolved in a mixture of $\mathrm{CH}_{3} \mathrm{CN} /$ water $(40 \mathrm{~mL} / 10 \mathrm{~mL})$ and refluxed for $6 \mathrm{~h}$. During this period a colorless clear solution was formed, which was kept for two days and led to the formation of the colorless crystalline compound. This colorless crystalline compound was filtered followed by washing with acetonitrile and dried under vacuum at $50{ }^{\circ} \mathrm{C}$ for $3 \mathrm{~h}$ to give $\mathbf{4 a}$ as colorless compound. Single crystals suitable for X-ray diffraction studies were grown by slow evaporation from a $\mathrm{CH}_{3} \mathrm{CN} /$ water (4/1) mixture solution of $\mathbf{4 a}$ at room temperature. Yield: 2.85 g, 48\%. M.p.: 102$104{ }^{\circ} \mathrm{C} .{ }^{1} \mathrm{H}$ NMR $\left(25{ }^{\circ} \mathrm{C}, 300 \mathrm{MHz}, \mathrm{CDCl}_{3}\right): \delta=8.78$ (s, br, 1H, P-OH), 4.10-4.06 (m, 2H, H1), 2.27-2.15 (m, 4H), 1.67-1.63 (m, 4H), 1.40-1.29 (m, 4H), 1.20$1.12(\mathrm{~m}, 2 \mathrm{H}), 1.0-0.97(\mathrm{~m}, 2 \mathrm{H}), 0.92-0.88(\mathrm{~m}, 14 \mathrm{H})$, 0.81-0.79 (m, 6H) ppm. ${ }^{13} \mathrm{C}\left\{{ }^{1} \mathrm{H}\right\}$ NMR $\left(25^{\circ} \mathrm{C}\right.$, $\left.75.431 \mathrm{MHz}, \mathrm{CDCl}_{3}\right): \delta=78.99(\mathrm{C} 1), 78.90(\mathrm{C} 1)$, 48.56, 48.47, 42.39 (C6), $34.09\left(\mathrm{CH}_{2}\right), 31.61,30.95$, $25.42\left(\mathrm{CH}\left(\mathrm{CH}_{3}\right)_{2}\right), 22.89\left(\mathrm{CH}_{2}\right), 22.01\left(\mathrm{CH}_{3}\right), 20.97$ $\left(\mathrm{CH}_{3}\right), \quad 15.74\left(\mathrm{CH}_{3}\right)$ ppm. ${ }^{31} \mathrm{P}\left\{{ }^{1} \mathrm{H}\right\}$ NMR $\left(25{ }^{\circ} \mathrm{C}\right.$, $\left.121.442 \mathrm{MHz}, \mathrm{CDCl}_{3}\right): \delta=0.55 \mathrm{ppm}$. FT-IR $(\mathrm{KBr}$ pellet, $\mathrm{cm}^{-1}$ ): $\bar{v}=3417$ (m, br), 2954 (s), 2927 (s), 2870 (m), 2722 (w), 2385 (w), 1639 (w), 1456 (s), 1387 (m), 1371 (m), 1349 (w), 1232 (w), 1180 (m), $1154(\mathrm{~m}), 1061$ (s), 1014 (s), 977 (s), 928 (m), 898 (m), $835(\mathrm{~m}), 818$ (w), $801(\mathrm{w}), 594(\mathrm{w}), 566(\mathrm{w}), 552$ (m). ESI-MS: Calcd $(\mathrm{m} / \mathrm{z})$ for $\left[\mathrm{C}_{20} \mathrm{H}_{39} \mathrm{O}_{4} \mathrm{P}+\mathrm{Na}^{+}\right]^{+}$: 397.2478; found: 397.2489 . 
$2.2 j$ Synthesis of $\mathbf{4 b} ;(+) \boldsymbol{M t}_{2} \boldsymbol{P}(\boldsymbol{O}) \boldsymbol{O H}$ Compound $\mathbf{4 b}$ was prepared starting from $(+)$-menthol as colorless crystals following the same procedure as described above for the synthesis of 4a. Quantities: $(+)$-menthol (5.0 g, $32 \mathrm{mmol}), n \mathrm{BuLi}(1.6 \mathrm{M}$ in hexane, $20 \mathrm{~mL}, 32$ $\mathrm{mmol}), \mathrm{POCl}_{3}(1.5 \mathrm{~mL}, 16 \mathrm{mmol})$, THF $(10 \mathrm{~mL})$, diethyl ether $(10 \mathrm{~mL})$. Single crystals suitable for Xray diffraction studies were grown by slow evaporation from a $\mathrm{CH}_{3} \mathrm{CN} /$ water (4/1) mixture solution of $\mathbf{4 b}$ at room temperature. Yield: 2.72 g, 45\%. M.p.: 108$110{ }^{\circ} \mathrm{C} .{ }^{1} \mathrm{H}$ NMR $\left(25{ }^{\circ} \mathrm{C}, 300 \mathrm{MHz}, \mathrm{CDCl}_{3}\right): \delta=9.24$ (s, br, 1H, P-OH), 4.10-4.06 (m, 2H, H1), 2.27-2.15 (m, 4H), 1.67-1.63 (m, 4H), 1.39-1.29 (m, 4H), 1.20$1.08(\mathrm{~m}, 2 \mathrm{H}), 1.0-0.97(\mathrm{~m}, 2 \mathrm{H}), 0.92-0.88(\mathrm{~m}, 14 \mathrm{H})$, 0.81-0.79 (m, 6H) ppm. ${ }^{13} \mathrm{C}\left\{{ }^{1} \mathrm{H}\right\}$ NMR $\left(25^{\circ} \mathrm{C}\right.$, $\left.75.431 \mathrm{MHz}, \mathrm{CDCl}_{3}\right): \delta=78.98(\mathrm{C} 1), 78.90(\mathrm{C} 1)$, 48.57, 48.47, 42.40 (C6), $34.10\left(\mathrm{CH}_{2}\right), 31.61,25.43$ $\left(\mathrm{CH}\left(\mathrm{CH}_{3}\right)_{2}\right), 22.90\left(\mathrm{CH}_{2}\right), 22.0\left(\mathrm{CH}_{3}\right), 20.96\left(\mathrm{CH}_{3}\right)$, $15.75\left(\mathrm{CH}_{3}\right)$ ppm. ${ }^{31} \mathrm{P}\left\{{ }^{1} \mathrm{H}\right\}$ NMR $\left(25^{\circ} \mathrm{C}, 121.442\right.$ $\mathrm{MHz}, \mathrm{CDCl}_{3}$ ): $\delta=0.55 \mathrm{ppm}$. FT-IR ( $\mathrm{KBr}$ pellet, $\left.\mathrm{cm}^{-1}\right): \bar{v}=3420(\mathrm{~m}, \mathrm{br}), 2954(\mathrm{~s}), 2927(\mathrm{~s}), 2870(\mathrm{~m})$, 2722 (w), 2385 (w), 1654 (m), 1456 (s), 1387 (m), 1371 (m), 1349 (w), 1231 (s), 1180 (m), $1154(\mathrm{~m})$, 1061 (s), 1014 (s), 977 (s), 928 (m), 898 (m), 835 (m), 818 (w), $801(\mathrm{w}), 772(\mathrm{w}), 594(\mathrm{w}), 566(\mathrm{~s}), 552(\mathrm{~s})$, 506 (w). ESI-MS: Calcd $(\mathrm{m} / \mathrm{z})$ for $\left[\mathrm{C}_{20} \mathrm{H}_{39} \mathrm{O}_{4} \mathrm{P}+\right.$ $\left.\mathrm{Na}^{+}\right]^{+}$: 397.2478; found: 397.2489.

2.2k Synthesis of $\mathbf{4 c} ;(+)-\mathbf{M t}(-)-\mathbf{M t P}(\mathrm{O}) \mathrm{OH} n \mathrm{BuLi}(1.6$ $\mathrm{M}$ in hexane, $20 \mathrm{~mL}, 32 \mathrm{mmol}$ ) was added dropwise to a solution of (+)-menthol $(5.0 \mathrm{~g}, 32 \mathrm{mmol}$, in $10 \mathrm{~mL}$ of dry diethyl ether) at $-78{ }^{\circ} \mathrm{C}$ resulting in the formation of a colorless suspension. Afterwards it was stirred for an additional $10 \mathrm{~min}$ at $-78^{\circ} \mathrm{C}$, the suspension was added dropwise to a solution containing $\mathrm{POCl}_{3}(9 \mathrm{~mL}, 96 \mathrm{mmol}$, in $30 \mathrm{~mL}$ of dry diethyl ether) at $-78^{\circ} \mathrm{C}$ and stirring was continued for overnight. All volatiles were removed under vacuum affording a colorless oily compound of $\mathrm{RO}-\mathrm{POCl}_{2} ; \mathrm{R}=(+)-\mathrm{Mt}$ with $\mathrm{LiCl}$. In another Schlenk flask, $n \mathrm{BuLi}(1.6 \mathrm{M}$ in hexane, $20 \mathrm{~mL}, 32 \mathrm{mmol}$ ) was added dropwise to a solution of (-)-menthol $(5.0 \mathrm{~g}, 32 \mathrm{mmol}$, in $10 \mathrm{~mL}$ of dry diethyl ether) at $-78{ }^{\circ} \mathrm{C}$. After completion of addition, the reaction mixture was stirred for an additional $10 \mathrm{~min}$ at $-78{ }^{\circ} \mathrm{C}$ resulting in the formation of a colorless suspension of $\mathrm{R}^{\prime} \mathrm{O}^{-} \mathrm{Li}^{+} ; \mathrm{R}=(-)$-Mt. This colorless suspension of $\mathrm{R}^{\prime} \mathrm{O}^{-} \mathrm{Li}^{+}$was added to a stirred solution of RO-POCl 2 in THF $(20 \mathrm{~mL})$ at $-78^{\circ} \mathrm{C}$ by cannula within $1 \mathrm{~h}$. Then the reaction mixture was slowly allowed to reach room temperature and stirring was continued for overnight. During this period a colorless suspension was formed, and then the volatiles were removed under vacuum affording a colorless oily compound of $(\mathrm{RO})\left(\mathrm{R}^{\prime} \mathrm{O}\right) \mathrm{POCl}$ with $\mathrm{LiCl}$. The resulting reaction mixture was dissolved in a mixture of $\mathrm{CH}_{3} \mathrm{CN} /$ water $(80 \mathrm{~mL} / 20 \mathrm{~mL})$ and refluxed for $6 \mathrm{~h}$. During this period a colorless clear solution was formed, which was kept for two days and lead to the formation of a colorless crystalline compound. This colorless crystalline compound was filtered followed by washing with acetonitrile and drying under vacuum at $50{ }^{\circ} \mathrm{C}$ for $3 \mathrm{~h}$ to give $\mathbf{4 c}$ as colorless compound. Single crystals suitable for $\mathrm{X}$-ray diffraction studies were grown by slow evaporation from a $\mathrm{CH}_{3} \mathrm{CN}$ /water (4/1) mixture solution of $\mathbf{4} \mathbf{c}$ at room temperature. Yield: 1.92 g, $16 \%$. M.p.: $118-120{ }^{\circ} \mathrm{C} .{ }^{1} \mathrm{H}$ NMR $\left(25^{\circ} \mathrm{C}, 300 \mathrm{MHz}, \mathrm{CDCl} 3\right): \delta=8.75$ (s, br, $1 \mathrm{H}, \mathrm{P}-$ $\mathrm{OH}), 4.15-4.04$ (m, 2H, H1), 2.27-2.13 (m, 4H), 1.67$1.63(\mathrm{~m}, 4 \mathrm{H}), 1.43-1.29(\mathrm{~m}, 4 \mathrm{H}), 1.20-1.08(\mathrm{~m}, 2 \mathrm{H})$, $1.01-0.97(\mathrm{~m}, 2 \mathrm{H}), 0.93-0.89(\mathrm{~m}, 14 \mathrm{H}), 0.82-0.79(\mathrm{~m}$, $6 \mathrm{H})$ ppm. ${ }^{13} \mathrm{C}\left\{{ }^{1} \mathrm{H}\right\} \quad \mathrm{NMR} \quad\left(25^{\circ} \mathrm{C}, 75.431 \mathrm{MHz}\right.$, $\left.\mathrm{CDCl}_{3}\right): \delta=79.09(\mathrm{C} 1), 79.0(\mathrm{C} 1), 48.52,48.43$, 42.40 (C6), $34.09\left(\mathrm{CH}_{2}\right), 31.6,25.42\left(\mathrm{CH}\left(\mathrm{CH}_{3}\right)_{2}\right)$, $22.92\left(\mathrm{CH}_{2}\right), 22.02\left(\mathrm{CH}_{3}\right), 20.96\left(\mathrm{CH}_{3}\right), 15.76\left(\mathrm{CH}_{3}\right)$ ppm. ${ }^{31} \mathrm{P}\left\{{ }^{1} \mathrm{H}\right\}$ NMR $\left(25^{\circ} \mathrm{C}, 121.442 \mathrm{MHz}, \mathrm{CDCl}_{3}\right)$ : $\delta=0.53$ ppm. FT-IR $\left(\mathrm{KBr}\right.$ pellet, $\left.\mathrm{cm}^{-1}\right): \bar{v}=3414$ (m, br), 2954 (s), 2925 (s), 2868 (s), 2656 (w), 2383 (w), $1684(\mathrm{w}), 1453$ (s), 1385 (m), 1368 (m), 1346 (w), 1219 (s), 1180 (m), 1159 (m), 1079 (m), 1061 (s), 1020 (s), 976 (s), 927 (m), $896(\mathrm{~m}), 835$ (m), $814(\mathrm{w})$, $799(\mathrm{w}), 771(\mathrm{w}), 593(\mathrm{w}), 557(\mathrm{~m}), 541(\mathrm{~m}), 506(\mathrm{w})$. ESI-MS: Calcd $(\mathrm{m} / \mathrm{z})$ for $\left[\mathrm{C}_{20} \mathrm{H}_{39} \mathrm{O}_{4} \mathrm{P}+\mathrm{Na}^{+}\right]^{+}$: 397.2478; found: 397.2487 .

\section{$2.3 X$-ray crystallography}

Single-crystal X-ray diffraction data of 1.DMF, 1.MeOH, 1.iPrOH (solvent of crystallization: acetone, acetonitrile and iso-propanol), 1.t $\mathbf{B u O H}$, 2.iPr $\mathbf{P r}_{2} \mathbf{N E t}, 2 \cdot \mathrm{NHC}^{i \operatorname{Pr} 2 \mathrm{Me} 2}, \mathbf{3}, \mathbf{4 a}, 4 \mathrm{~b}$ and $4 \mathrm{c}$ were collected on a Rigaku XtaLAB X-ray Diffractometer system equipped with a CCD area detector and operated at $30 \mathrm{~W}$ power $(50 \mathrm{kV}, 0.6 \mathrm{~mA})$ to generate $\mathrm{Mo}$ $\mathrm{K} \alpha$ radiation $(\lambda=0.71073 \AA$ ) at $120(2) \mathrm{K}$. Singlecrystal X-ray diffraction data of 1.EtOH and 1.iPrOH (solvent of crystallization: iso-propanol) were collected at low temperature $(170 \mathrm{~K})$ on a STOE-IPDS II diffractometer equipped with a normal-focus, $2.4 \mathrm{~kW}$, sealed tube X-Ray source with graphite-mono-chromated Mo $\mathrm{K} \alpha$ radiation $(\lambda=0.71073 \AA)$. The integration of diffraction profiles was performed with the program XArea; numerical absorption correction was carried out with the programs X-shape and X-red32; all from $\mathrm{STOE}^{\odot}$. The structures were solved with the SHELXT structure solution program using Intrinsic 
Phasing and refined with the SHELXL refinement package using Least Squares minimisation in the Olex-2 software. ${ }^{39-43}$ All non-hydrogen atoms were refined with anisotropic thermal parameters. All hydrogen atoms were placed in geometrically calculated positions or found in the Fourier difference map and included in the refinement process using a riding model. Crystal data and structure refinement of all these compounds are given in Tables S5 and S6 (Supplementary Information).

\section{Results and Discussion}

We have synthesized 1.Et $\mathbf{t}_{\mathbf{2}} \mathrm{O}$ according to the procedure shown in Scheme 1. ${ }^{37}$ Subsequently, compound $\mathbf{1} \cdot \mathbf{E t}_{2} \mathbf{O}$ was crystallized using different solvents capable of hydrogen bonding such as DMF, $\mathrm{CH}_{3} \mathrm{OH}$, $\mathrm{EtOH}$, and $i \mathrm{PrOH}$ as well as from the mixtures of $i \mathrm{PrOH} / \mathrm{CH}_{3} \mathrm{COCH}_{3} / \mathrm{CH}_{3} \mathrm{CN}$ and $t \mathrm{BuOH} / \mathrm{CH}_{3} \mathrm{COCH}_{3} /$ $\mathrm{CH}_{3} \mathrm{CN}$ solvents to study the H-bonding interaction. Crystallization of the phosphate diester in $\mathrm{CH}_{3}$ $\mathrm{COCH}_{3}, \mathrm{CH}_{2} \mathrm{Cl}_{2}, \mathrm{CHCl}_{3}$ or DMSO did not succeed. Analysis of the single crystals of 1.DMF obtained from $\mathrm{DMF}$ indicates two types of $\mathrm{H}$-bond between $\mathrm{P}-$ $\mathrm{O}-\mathrm{H} \cdots \mathrm{O}=\mathrm{CH}$ and $\mathrm{P}=\mathrm{O} \cdots \mathrm{H}-\mathrm{C}=\mathrm{O}$ moieties. This results in the stabilization of the monomer in the solid-state. On the other hand, analysis of crystals obtained from $\mathrm{CH}_{3} \mathrm{OH}, \mathrm{EtOH}, i \mathrm{PrOH} / \mathrm{CH}_{3} \mathrm{COCH}_{3} / \mathrm{CH}_{3} \mathrm{CN}$ indicated the formation of an alcohol-assisted dimer (Figure 1). The important bond distances and angles of these compounds are given in Tables S1 and S2 (Supplementary Information).

The molecular structures of the phosphate diesters, 1.DMF, 1.MeOH, 1.EtOH and 1.iPrOH (solvent of crystallization: acetone, acetonitrile and iso-propanol) reveal three types of $\mathrm{P}-\mathrm{O}$ bond distances (Tables $\mathrm{S} 1$ and S2, Supplementary Information). The shortest distance is $1.469(3) \AA \quad$ (1.DMF), $1.471(11) \AA$ (1.MeOH), 1.471(4) $\AA$ (1·EtOH) and 1.473(11) $\AA$ (1-iPrOH) which is consistent with the $\mathrm{P}=\mathrm{O}$ distance of similar compounds. A total of six $\mathrm{O}-\mathrm{P}-\mathrm{O}$ angles were found in each structure: $109.05(17)^{\circ}$, $107.49(16)^{\circ}, \quad 95.06(15)^{\circ}, \quad 113.30(17)^{\circ}, 115.20(17)^{\circ}$, 114.82(17) $)^{\circ} \quad(\mathbf{1} \cdot \mathbf{D M F}), \quad 103.64(6)^{\circ}, \quad 105.31(6)^{\circ}$,

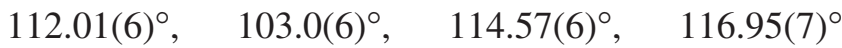
$(\mathbf{1} \cdot \mathbf{M e O H}) ; \quad 116.95(4)^{\circ}, \quad 112.01(2)^{\circ}, \quad 114.28(23)^{\circ}$, $105.71(24)^{\circ}, 103.14(3)^{\circ}, 103.40(33)^{\circ}(\mathbf{1} \cdot \mathbf{E t O H})$ and $102.78(6)^{\circ}, \quad 103.45(6)^{\circ}, \quad 115.12(6)^{\circ}, \quad 105.67(6)^{\circ}$, $112.22(6)^{\circ}, \quad 116.21(7)^{\circ}(\mathbf{1} \cdot \mathbf{i P r O H})$. In all these instances, the central prochiral phosphorus centre is present in a distorted tetrahedral geometry. The

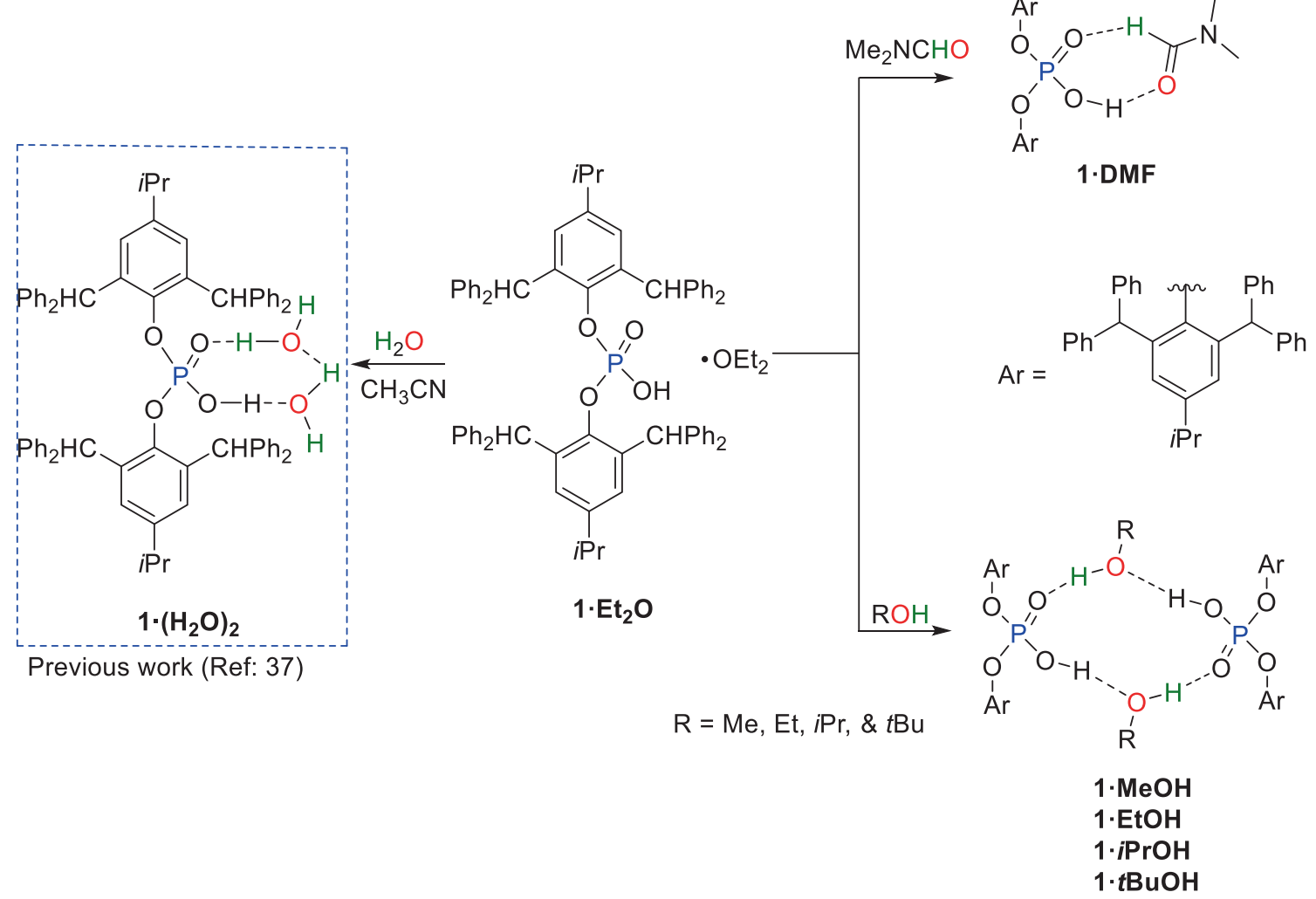

Scheme 1. Synthesis of 1.DMF, 1·MeOH, 1.EtOH, 1.iPrOH and 1.tBuOH. 


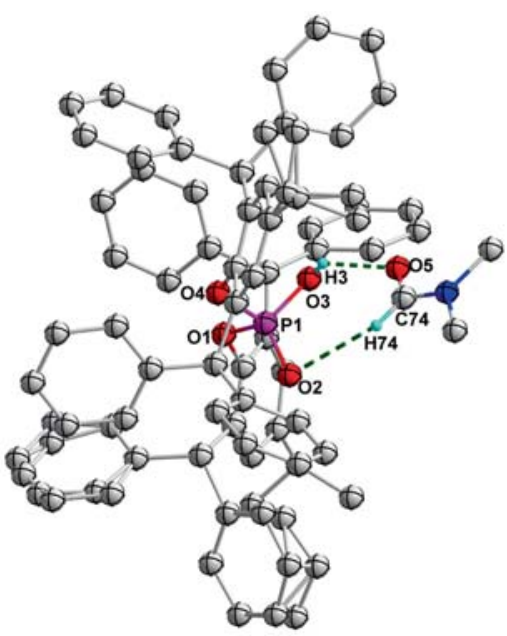

(a)

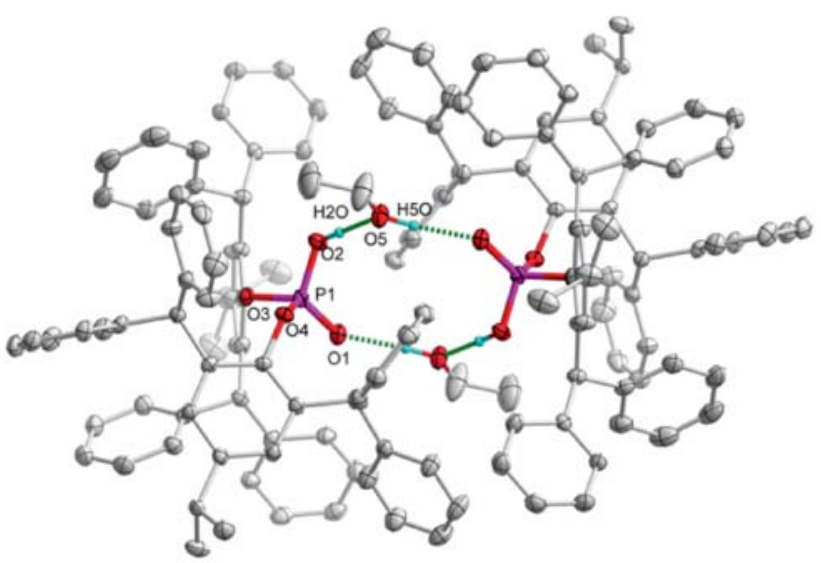

(c)

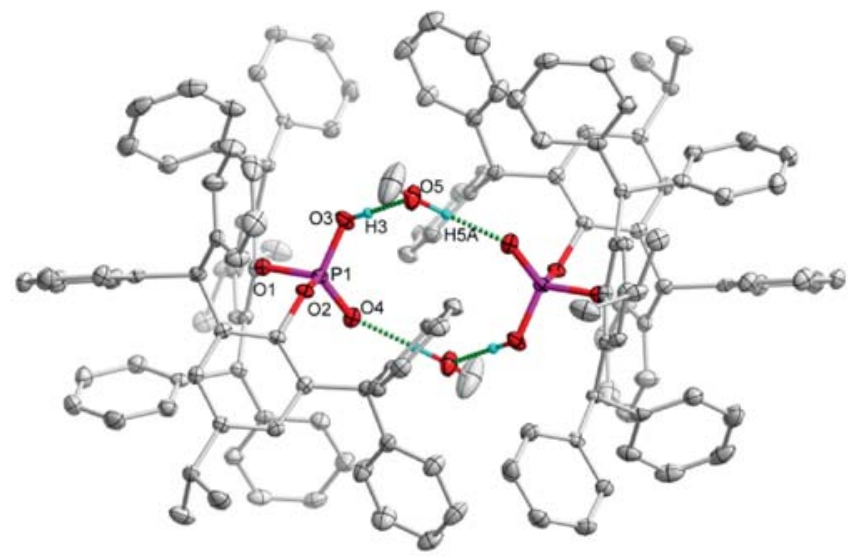

(b)

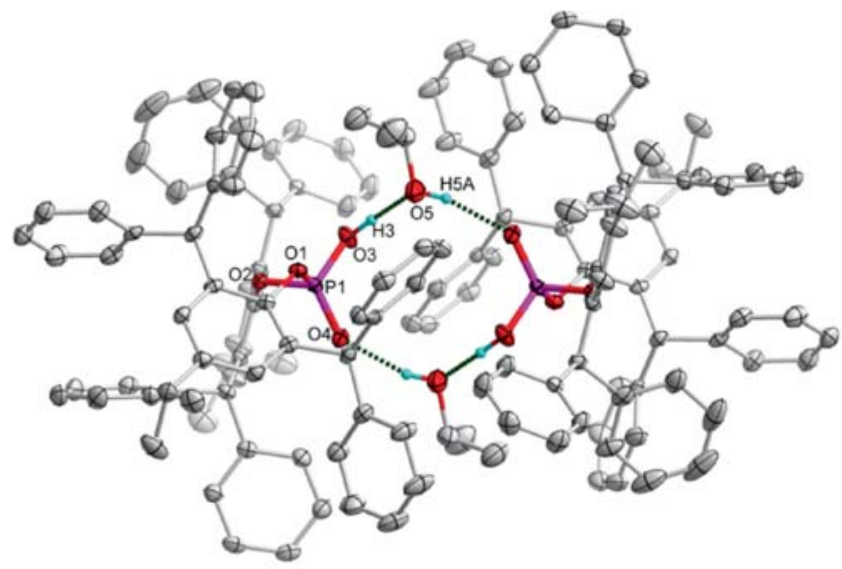

(d)

Figure 1. H-bonded molecular structures of (a) 1·DMF, (b) 1·MeOH, (c) 1·EtOH and (d) 1·iPrOH; all hydrogen atoms except $\mathrm{O}-\mathrm{H}, \mathrm{C}-\mathrm{H}$ of DMF and one disordered solvent molecule in 1.DMF were omitted for clarity reasons and thermal ellipsoids represent the $50 \%$ probability level in all cases.

molecular structure of 1.DMF shows a non-planar seven-membered ring (Figure 1), and in the case of 1-MeOH, 1.EtOH and 1.iPrOH non-planar twelvemembered rings (Figure 1). The crystal structure of 1.DMF also shows two types of intermolecular hydrogen-bonds $\quad(\mathrm{P}-\mathrm{OH} \cdots \mathrm{O}=\mathrm{C}=1.843(7) \AA \quad /$ $\mathrm{P}=\mathrm{O} \cdots \mathrm{H}-\mathrm{C}=2.733 \AA$ ) with an $\mathrm{O} \cdots \mathrm{O}$ separation of only 2.418(4) $\AA$. On the other hand, crystal structures of 1.MeOH, 1.EtOH and 1.iPrOH reveal intermolecular hydrogen-bonding between one molecule of phosphate diester and one molecule of alcohol $(\mathrm{P}-$ $\mathrm{OH} \cdots \mathrm{OH}-\mathrm{R} / \mathrm{P}=\mathrm{O} \cdots \mathrm{H}-\mathrm{OR})$ via two short $\mathrm{O}-\mathrm{H} \cdots \mathrm{O}$ bonds $\quad[\mathrm{P}-\mathrm{OH} \cdots \mathrm{OH}-\mathrm{R}=1.584(3) \AA \quad(\mathbf{1} \cdot \mathbf{M e O H})$, $1.532(7) \AA \quad(\mathbf{1} \cdot \mathbf{E t O H})$ and $1.51(3) \AA \quad(\mathbf{1} \cdot \mathbf{i P r O H})$; $\mathrm{P}=\mathrm{O} \cdots \mathrm{H}-\mathrm{OR}=1.75(3) \AA \quad(\mathbf{1} \cdot \mathbf{M e O H}), \quad 1.762(7) \AA$ (1.EtOH) and $1.91 \AA(\mathbf{1} \cdot \mathbf{i P r O H})]$ with $\mathrm{O} \cdots \mathrm{O}$ separations of $2.435(16) \AA / 2.671(16) \AA \quad(\mathbf{1} \cdot \mathbf{M e O H})$, 2.433(11) $\AA / 2.652(10) \AA$ (1.EtOH) and 2.413(18) $\AA /$
2.703(17)Å (1-iPrOH). 1·iPrOH when crystallized in pure iso-propanol solvent results in severe disorder in the iso-propanol and therefore the H-bonding interactions are not discussed. The metric parameters of 1.iPrOH and 1.t $\mathbf{B u O H}$ are given in Table S2, Supplementary Information.

Previously we reported the reaction of $\mathbf{1} \cdot \mathbf{E t}_{2} \mathbf{O}$ with $\mathrm{Et}_{3} \mathrm{~N}$ affording anionic phosphate diester, $\mathbf{2} \cdot \mathbf{E t}_{\mathbf{3}} \mathbf{N}{ }^{37}$ Subsequently, we also performed related reactions of $\mathbf{1} \cdot \mathbf{E t}_{2} \mathrm{O}$ with one equivalent of $i \operatorname{Pr}_{2} \mathrm{NEt}$ or $\mathrm{NHC}^{i \mathrm{Pr} 2 \mathrm{Me} 2}$, resulting in the formation of colourless compounds 2.iPr $\mathbf{P r}_{\mathbf{2}} \mathbf{N E t}$ and $\mathbf{2} \cdot \mathbf{N H C}{ }^{\mathbf{i P r} \mathbf{P e} 2}$, respectively. The characterization of $\mathbf{2} \cdot \mathbf{i} \mathbf{P r}_{2} \mathbf{N E t}$ and $\mathbf{2} \cdot \mathbf{N H C}^{i \mathbf{P r} 2 \mathrm{Me} 2}$ in solution was carried out by NMR and ESI-MS methods. The most prominent features were the appearance of resonances of $\mathrm{N}-\mathrm{H}$ in $\mathbf{2} \cdot \mathbf{i} \mathbf{P r}_{\mathbf{2}} \mathbf{N E t}$ or $\mathrm{C} 2-\mathrm{H}$ in 2.NHC ${ }^{i \mathbf{P r 2 M e} 2}$ in the deshielded region $(11.25 \mathrm{ppm}$ in $\mathrm{CDCl}_{3}$ for $\mathbf{2} \cdot \mathbf{i} \mathbf{P r}_{2} \mathbf{N E t}$ and $10.54 \mathrm{ppm}$ in $\mathrm{CDCl}_{3}$ for 


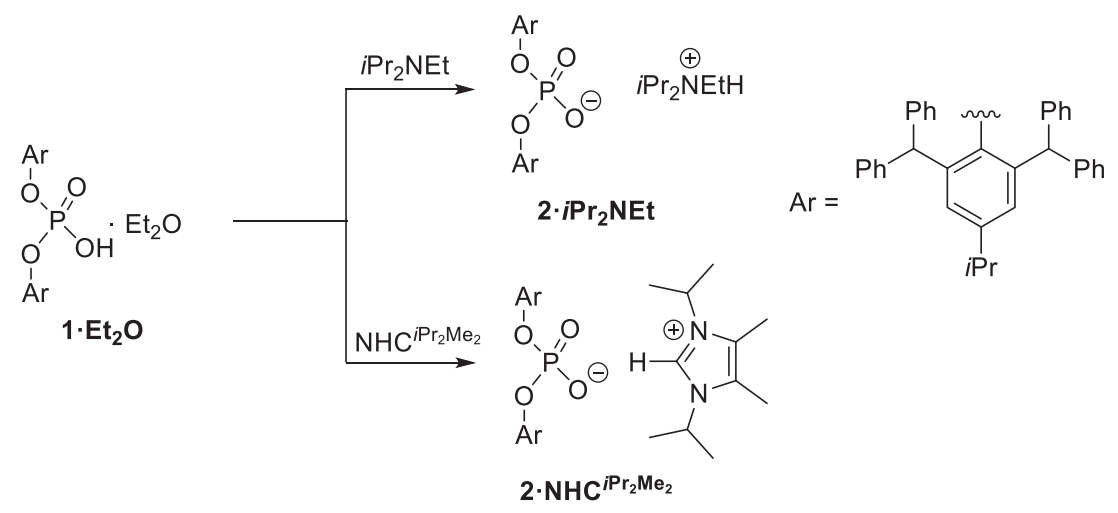

Scheme 2. Synthesis of $2 \cdot \boldsymbol{i} \mathbf{P r}_{2} \mathbf{N E t}$ and $2 \cdot \mathbf{N H C}^{i \operatorname{Pr} 2 \mathrm{Me} 2}$.

Table 1. ${ }^{31} \mathrm{P}\left\{{ }^{1} \mathrm{H}\right\}$ NMR and selected ${ }^{1} \mathrm{H}$ NMR data of newly synthesized compounds.

\begin{tabular}{lcc}
\hline Compound & $\delta \begin{array}{c}{ }^{31} \mathrm{P}\left\{{ }^{1} \mathrm{H}\right\} \text { NMR } \\
(\mathrm{ppm})\end{array}$ & $\begin{array}{c}{ }^{1} \mathrm{HNMR} \text { of P-OH } \\
(\mathrm{ppm})\end{array}$ \\
\hline $\mathbf{2} \cdot \mathbf{i} \mathbf{P r}_{2} \mathbf{N E t}$ & -11.0 & \\
$\mathbf{2} \cdot \mathbf{N H C}{ }^{\text {Pr2Me2 }}$ & -9.2 & \\
$\mathbf{3}$ & -10.6 & \\
$\mathbf{4 a}$ & 0.55 & 8.78 \\
$\mathbf{4 b}$ & 0.55 & 9.24 \\
$\mathbf{4 c}$ & 0.53 & 8.75 \\
\hline
\end{tabular}

All NMR spectra were measured using $\mathrm{CDCl}_{3}$.

2.NHC ${ }^{\text {iPr2Me2 }}$ ) (Scheme 2, Figures S1 and S4, Supplementary Information). The ${ }^{31} \mathrm{P}\left\{{ }^{1} \mathrm{H}\right\}$ solution NMR spectra of $\mathbf{2} \cdot \mathbf{i P r}_{2} \mathbf{N E t}$ or $\mathbf{2} \cdot \mathbf{N H C}{ }^{i \mathbf{P r 2 M e} 2}$ show each a singlet at $\delta=-11.0$ or $-9.2 \mathrm{ppm}$ suggesting the presence of only one type of phosphorus centre in the dissolved state (Table 1). The base peak in the ESIMS spectrum for $\mathbf{2} \cdot \mathbf{N H C}^{i \mathbf{P r 2 M e} 2}$ recorded in positive ion mode corresponds to the imidazolium cation. Furthermore, $\mathbf{2} \cdot \mathbf{i} \mathbf{P r}_{2} \mathbf{N E t}$ and $\mathbf{2} \cdot \mathbf{N H C} \mathbf{C}^{\mathbf{i P r 2 M e} 2}$ were characterized in their solid-state by single-crystal $\mathrm{X}$-ray diffraction analysis.

The asymmetric units of $\mathbf{2} \cdot \boldsymbol{i} \mathbf{P r}_{\mathbf{2}} \mathbf{N E t}$ and 2.NHC ${ }^{i \mathbf{P r 2 M e 2}}$ are shown in Figure 2. The crystallographic parameters for these compounds are given in Table S6 (Supplementary Information). The negative charge of the phosphate diester ligand in $\mathbf{2} \cdot \mathbf{i} \mathbf{P r}_{2} \mathbf{N E t}$ and $\mathbf{2} \cdot \mathbf{N H C} \mathbf{C}^{i \mathbf{P r} \mathbf{M e} 2}$ is delocalized as confirmed from the $\mathrm{P}-\mathrm{O}$ distances [P1-O1 1.622(7), P1-O2 1.618(6), P1-O3 1.490(7), P1-O4 1.467(3) in 2.iPr 2 NEt and $\mathrm{P} 1-\mathrm{O} 1$ 1.625(1), P1-O2 1.628(6), P1-O3 1.468(5), $\mathrm{P} 1-\mathrm{O} 4 \mathbf{1 . 4 8 6 ( 3 )}$ in $\mathbf{2} \cdot \mathbf{N H C}^{\mathbf{i P r 2 M e}}$ ]. The phosphorus atom exhibits a distorted tetrahedral geometry with the
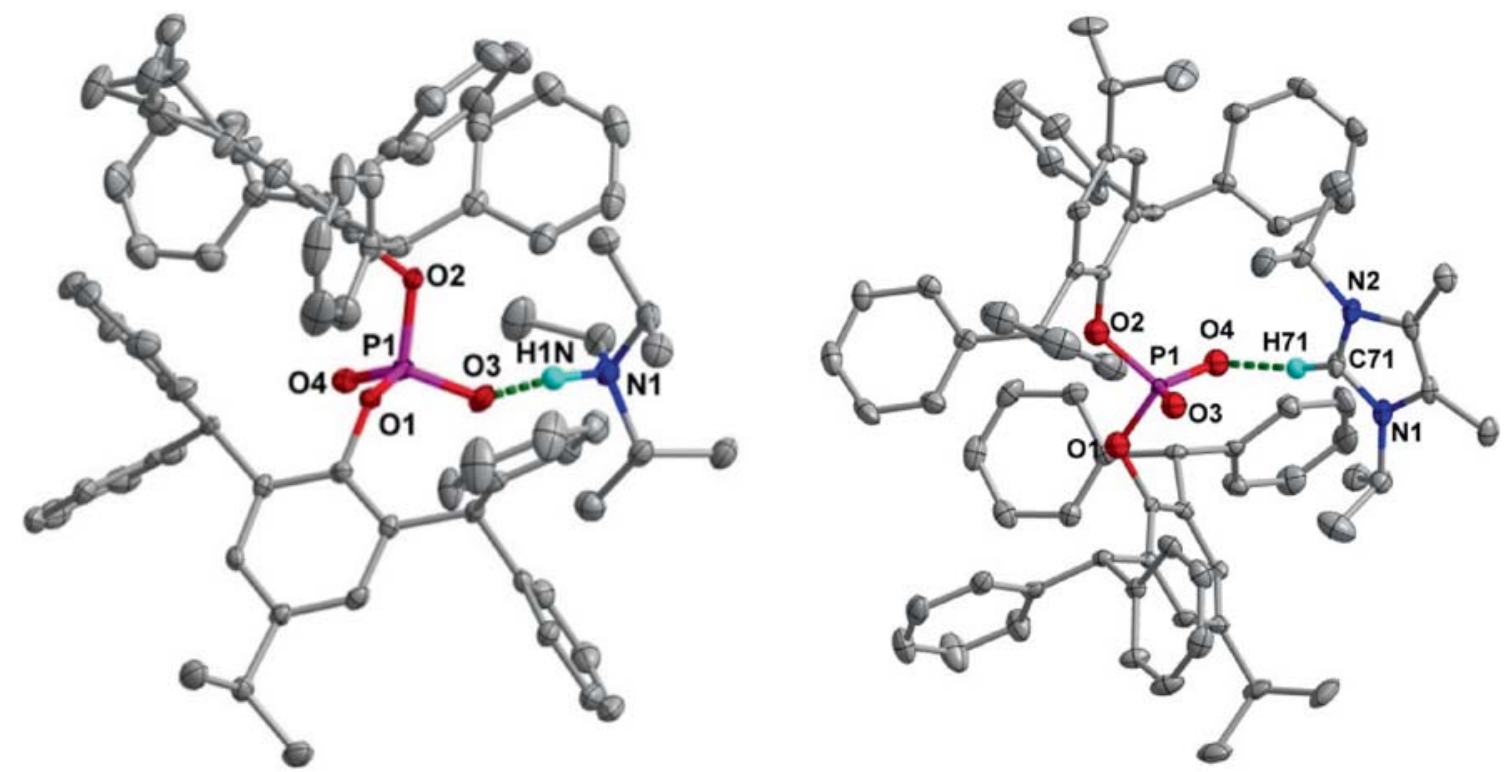

Figure 2. Molecular structures of $\mathbf{2} \cdot \mathbf{i} \mathbf{P r}_{2} \mathbf{N E t}$ and $\mathbf{2} \cdot \mathbf{N H C} \mathbf{C}^{\mathbf{P P r 2 M e} 2}$. Thermal ellipsoids are at the $50 \%$ probability level (hydrogen atoms except $\mathrm{N}-\mathrm{H}$ for $\mathbf{2} \cdot \mathbf{i} \mathbf{P r}_{2} \mathbf{N E t}$ and $\mathrm{C} 71-\mathrm{H} 71$ for $\mathbf{2} \cdot \mathbf{N H C} \mathbf{P r}^{\mathbf{P} \mathbf{M e} 2}$ are omitted for clarity). 


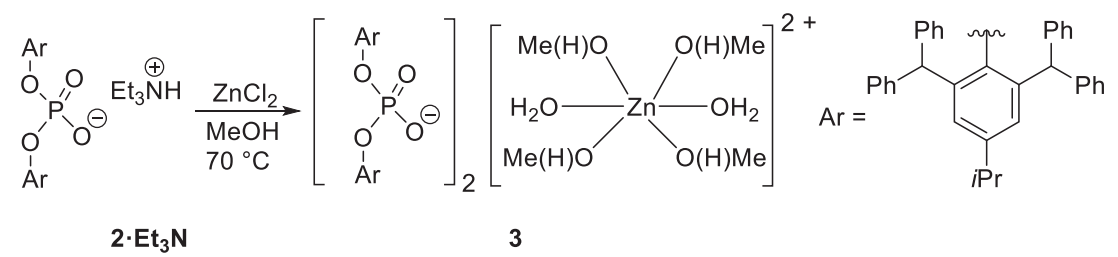

Scheme 3. Synthesis of 3.

angles varying from $98.09(9)^{\circ}-119.37(11)^{\circ}$ in $\mathbf{2} \cdot \mathbf{i} \mathbf{P r}_{2}$ NEt and $93.84(8)^{\circ}-118.47(10)^{\circ}$ in $\mathbf{2} \cdot \mathbf{N H C}^{i \mathbf{P r} \mathbf{M e} 2}$ (Table S3, Supplementary Information). Ethyl diisopropyl ammonium $\left(\mathbf{2} \cdot \mathbf{i} \mathbf{P r}_{\mathbf{2}} \mathbf{N E t}\right)$ and imidazolium $\left(\mathbf{2} \cdot \mathbf{N H C}^{i \mathrm{Pr2Me2}}\right)$ cations are involved in strong hydrogen bonding interaction with the anionic phosphate diester $\left[\mathrm{N} 1-\mathrm{H} 1 \mathrm{~N} \cdots \mathrm{O} 3,1.61(3) \AA\right.$ in $\mathbf{2} \cdot \mathbf{i} \mathbf{P r}_{\mathbf{2}} \mathbf{N E t}$ and C71-H71.O4 2.00(3) $⿱$ A in 2.NHC $\left.{ }^{\text {iPr2Me2 }}\right]$.

Subsequently, we were interested to obtain zincphosphate diester complex using $\mathbf{2} \cdot \mathbf{E t}_{\mathbf{3}} \mathbf{N}$ and $\mathrm{ZnCl}_{2}$. However, the reaction of $\mathbf{2} \cdot \mathbf{E t}_{\mathbf{3}} \mathbf{N}$ with $\mathrm{ZnCl}_{2}$ in methanol afforded the ion-pair $\mathbf{3}$ (Scheme 3). The ${ }^{31} \mathrm{P}\left\{{ }^{1} \mathrm{H}\right\}$ NMR spectrum of $\mathbf{3}$ revealed the presence of a singlet at $\delta=-10.6 \mathrm{ppm}$ suggesting the presence of only one type of phosphorus centre in the dissolved state (Table 1). In comparison to the ion pair $\mathbf{2} \cdot \mathbf{E t}_{\mathbf{3}}$ $\mathbf{N}(\delta=-9.8)$, the chemical shift for $\mathbf{3}$ appears in the upfield region.

The solid-state structure of $\mathbf{3}$ is shown in Figures 3 and S28, Supplementary Information. The crystallographic parameters are given in Table S6 (Supplementary Information). The asymmetric unit contains two anionic diester phosphates and the cationic hexacoordinated $\mathrm{Zn}(\mathrm{II})$ complex, $\left[\mathrm{Zn}\left(\mathrm{H}_{2} \mathrm{O}\right)_{2}\left(\mathrm{CH}_{3} \mathrm{OH}\right)_{4}\right]^{2+}$. The negative charge of the phosphate diester in $\mathbf{3}$ is delocalized as confirmed from the $\mathrm{P}-\mathrm{O}$ distances [P1-O1 1.611(3), P1-O2 1.603(2), P1-O3 1.486(3), $\mathrm{P} 1-\mathrm{O} 4$ 1.491(3) in 3] and the phosphorous atom is present in a distorted tetrahedral geometry with angles around phosphorus varying from $100.59(13)^{\circ}-$ $117.23(15)^{\circ}$ (Table S3, Supplementary Information). The coordinating methanol and methoxide ligands in the hexa-coordinated cationic moiety $\left[\mathrm{Zn}\left(\mathrm{H}_{2} \mathrm{O}\right)_{2}(-\right.$ $\left.\left.\mathrm{CH}_{3} \mathrm{OH}\right)_{4}\right]^{2+}$ and, however, disordered in two crystallographic special positions (Figure 3; only one part is shown) and therefore the metric parameters are not discussed.

Subsequently, in order to explore the assembly of chiral phosphate ligands, menthol-based compounds $\mathbf{4 a}, \mathbf{4 b}$ and $\mathbf{4 c}$ were prepared (Scheme 4). While hydrolysis of the symmetric phosphoryl dichlorides afforded $\mathbf{4 a}$ and $\mathbf{4 b}$, the corresponding hydrolysis of the unsymmetrical derivative afforded $\mathbf{4 c}$ (Scheme 4).

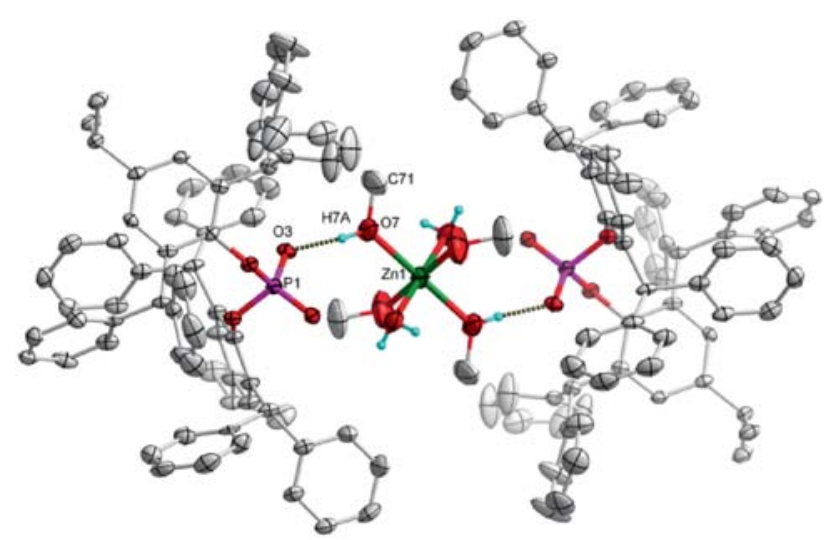

Figure 3. Molecular structure of $\mathbf{3}$ revealing the hydrogen bonding interactions. Thermal ellipsoids are at the $50 \%$ probability level (hydrogen atoms except $\mathrm{O}-\mathrm{H}$ are omitted for clarity).

The ${ }^{1}$ HNMR spectra of $\mathbf{4 a}, \mathbf{4 b}$ and $\mathbf{4 c}$ reveal the presence of a broad singlet in each corresponding to $\mathrm{PO} H$ at $\delta=8.78,9.24$ and $8.75 \mathrm{ppm}$, respectively (Table 1). The ${ }^{31} \mathrm{P}\left\{{ }^{1} \mathrm{H}\right\}$ NMR spectra of $\mathbf{4 a}, \mathbf{4 b}$ and $\mathbf{4 c}$ revealed singlets at $\delta=0.55,0.55$ and $0.53 \mathrm{ppm}$, respectively, which are upfield shifted compared to the resonances observed in the corresponding phosphoryldichlorides ( $\delta: 3.3 \mathrm{ppm}$ ) (Table 1$)$. Single crystals of $\mathbf{4 a}, \mathbf{4 b}$, and $\mathbf{4 c}$ were obtained by slow evaporation from $\mathrm{CH}_{3} \mathrm{CN}$ /water mixture solutions and were subjected to X-ray diffraction studies (Figure 4, Figures S29-S30, Supplementary Information).

The important bond distances and angles of $\mathbf{4 a}, \mathbf{4 b}$ and $\mathbf{4 c}$ are given in Table S4, Supplementary Information. The molecular structures of $\mathbf{4 a}, \mathbf{4 b}$ and $\mathbf{4 c}$, show three types of $\mathrm{P}-\mathrm{O}$ bond distances (Table S4, Supplementary Information). The shortest distance is 1.492(5) $\AA$ (4a), 1.489(4) $\AA$ (4b) and 1.495(1) $\AA$ (4c), which is consistent with the $\mathrm{P}=\mathrm{O}$ distance of known similar compounds. A total of six O-P-O angles were found in each structure: [101.9(3), 109.3(3), 110.4(3), 111.5(3), 107.2(3), 115.6(3) and 111.3(4) (4a)], [102.0(2), 106.9(3), 108.9(2), 116.0(3), 111.5(3) and 110.6(3) (4b)] and [103.40(6), 108.70(7), 112.26(6), 115.31(7), 109.50(6) and 106.99(7) (6c). The molecular structures of $\mathbf{4 a}$ and $\mathbf{4 c}$ reveal a non-planar eight- 


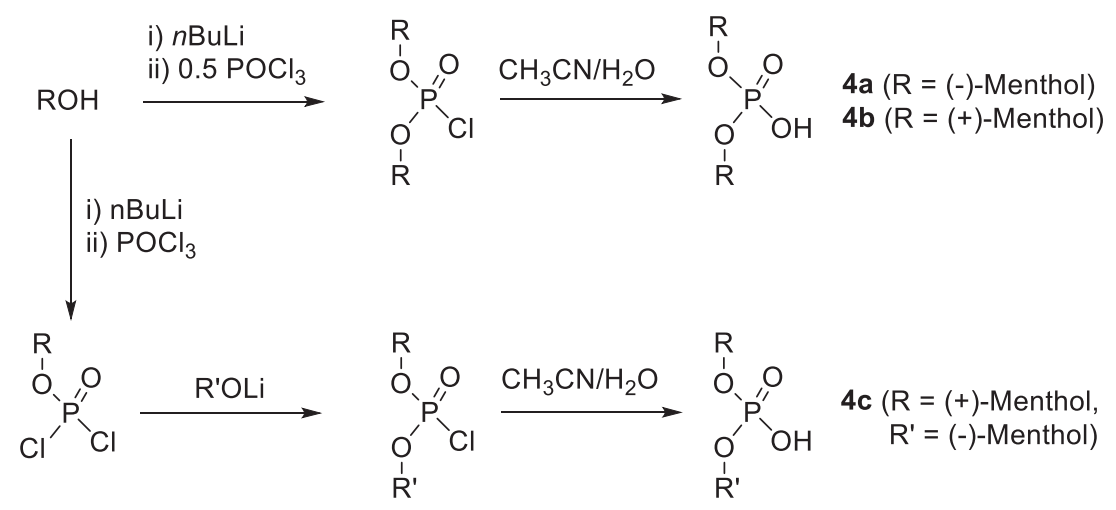

Scheme 4. Syntheses of $\mathbf{4 a}, \mathbf{4 b}$ and $\mathbf{4 c}$.

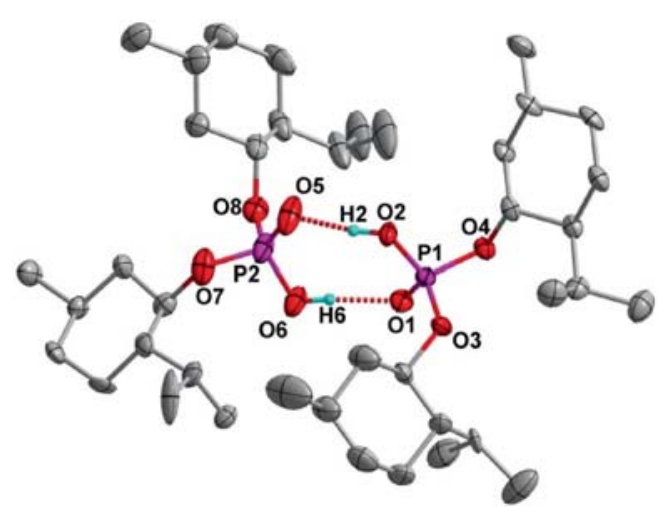

Figure 4. Molecular structure of 4a showing the hydrogen bonding interactions in the crystal. Thermal ellipsoids are at the $50 \%$ probability level (hydrogen atoms except $\mathrm{O}$ $\mathrm{H}$ are omitted for clarity).

membered ring (Figures 4 and S30, Supplementary Information) as a result of an intermolecular hydrogen-bonding network between the two molecules of phosphate diesters $(\mathrm{P}=\mathrm{O} / \mathrm{P}-\mathrm{OH})$ via two short $\mathrm{O}-\mathrm{H} \cdots \mathrm{O}$ bonds $[\mathrm{P}=\mathrm{O} \cdots \mathrm{HO}-\mathrm{P}=\{1.611(8) \AA$ and $1.700(5) \AA$ (4a) $\}$ and $1.779(3) \AA(\mathbf{4 c})]$ with a $\mathrm{O} \cdots \mathrm{O}$ separation of $\{2.472(5) \AA$ and $2.527(3) \AA(4 a)\}$, and 2.533(1) $\AA$ (4c)]. In the case of $\mathbf{4 b}$, these hydrogen-bonding interactions are missing; this can be attributed to the orientation of the hydroxide moiety of the $\mathrm{P}-\mathrm{OH}$ group which leads to the monomeric structure in the solid state.

\section{Conclusions}

In summary, we report the monomeric solid-state molecular structure of 2,6-(CHPh $)_{2}-4-i \operatorname{Pr}$-phenyl substituted phosphate diester as crystallized from DMF solvent. With alcohols as the crystallizing solvents, solvent-bridged dimeric structures are the result. The reactions of phosphate diesters with bases such as amine and NHC afforded ion-pairs which are present as monomers in the solid-state. We also obtained the anionic phosphate diester with a cationic $\mathrm{Zn}$ (II) complex as the counter cation. In addition, we have also synthesized phosphate diesters containing (-)-menthol, (+)-menthol and $(+) /(-)$-menthol substituents.

\section{Supplementary Information (SI)}

CCDC 1892137-1892148 contains the supplementary crystallographic data for 1.DMF, 1.MeOH, 1.EtOH, 1.iPrOH (Solvent of crystallization: iso-propanol), 1.iPrOH (Solvent of crystallization: acetone, acetonitrile and iso-propanol), 1.t $\mathbf{B u O H}$ (Solvent of crystallization: acetone, acetonitrile and tert-butanol), $2 \cdot \mathbf{i P r}_{2} \mathbf{N E t}$ and 2.NHC ${ }^{i \mathbf{P r 2 M e} 2}, \mathbf{3}, \mathbf{4 a}, \mathbf{4 b}$, and $\mathbf{4 c}$. These data can be obtained free of charge via http://www.ccdc.cam.ac.uk/ conts/retrieving.html, or from the Cambridge Crystallographic Data Centre, 12 Union Road, Cambridge CB2 1EZ, UK; fax: (+44) 1223-336-033; or e-mail: deposit@ccdc.cam.ac.uk. Supplementary data associated with this article is available at www.ias.ac.in/chemsci.

\section{Acknowledgements}

This work is supported by the TIFR Centre for Interdisciplinary Science Hyderabad, Hyderabad, India. V. G. acknowledges financial support from the Science and Engineering Research Board (SERB-NPDF) (Project No. PDF/2017/001902), Government of India. VC is thankful to the Department of Science and Technology, New Delhi, India, for National J. C. Bose fellowships.

\section{References}

1. Murugavel R, Choudhury A, Walawalkar M G, Pothiraja R and Rao C N R 2008 Metal Complexes of Organophosphate Esters and Open-Framework Metal Phosphates: Synthesis, Structure, Transformations, and Applications Chem. Rev. 1083549 
2. Roesky H W, Walawalkar M G and Murugavel R 2001 Is Water a Friend or Foe in Organometallic Chemistry? The Case of Group 13 Organometallic Compounds Acc. Chem. Res. 34201

3. Murugavel R, Walawalkar M G, Dan M, Roesky H W and Rao C N R 2004 Transformations of Molecules and Secondary Building Units to Materials: A Bottom-Up Approach Acc. Chem. Res. 37763

4. Guillou N, Gao Q, Forster P M, Chang J-S, Nogus M, Park S-E, Frey G and Cheetham 2001 A K, Nickel(ii) Phosphate VSB-5: A Magnetic Nanoporous Hydrogenation Catalyst with 24-Ring Tunnels Angew. Chem. 1132913

5. Walawalkar M G, Roesky H W and Murugavel R 1999 Molecular Phosphonate Cages: Model Compounds and Starting Materials for Phosphate Materials Acc. Chem. Res. 32117

6. Goura J and Chandrasekhar V 2015 Molecular Metal Phosphonates Chem. Rev. 1156854

7. Murugavel R, Kuppuswamy S, Gogoi N and Steiner A 2010 Assembling Discrete D4R Zeolite SBUs through Noncovalent Interactions. 3. Mediation by Butanols and 1,2-Bis(dimethylamino)ethane Inorg. Chem. 49 2153

8. Murugavel R, Kuppuswamy S, Maity A N and Singh M P 2009 Di-, Tri-, Tetra-, and Hexanuclear Copper(II) Mono-organophosphates: Structure and Nuclearity Dependence on the Choice of Phosphorus Substituents and Auxiliary N-Donor Ligands Inorg. Chem. 48183

9. Murugavel R and Shanmugan S 2008 Asymmetric Pentameric and Tetrameric Organooxotin Clusters: Insights into Their Formation through Partial Dearylation Organometallics 272784

10. Cheng W, Feng Z-Q and Tang J-M 2011 Bis(3,5dimethoxyphenyl)phosphinic acid Acta Crystallogr., Sect. E 67 o896

11. Székely G, Farkas V, Párkányi L, Tóth T, Hollósi M. and Huszthy P 2010 Crystal structures of crown ethers containing an alkyl diarylphosphinate or a diarylphosphinic acid unit Struct. Chem. 21277

12. Lyssenko K A, Grintselev-Knyazev G V and Antipin M Y 2002 Nature of the P-O bond in diphenylphosphonic acid: experimental charge density and electron localization function analysis Mendeleev Commun. 12 128

13. Fenske D, Mattes R, Löns J and Tebbe K-F 1973 Die Kristallstruktur von Diphenylphosphinsäure Chem. Ber. 1061139

14. Xu B, Zhu S-F, Zhang Z-C, Yu Z-X, Ma Y. and Zhou Q-L 2014 Highly enantioselective S-H bond insertion cooperatively catalyzed by dirhodium complexes and chiral spiro phosphoric acids Chem. Sci. 51442

15. Nayak S K, Chandrasekhar S and Row T N G 2008 1,1'-Bi-naphthalene-2,2'-diyl hydrogen phosphate Acta Crystallogr., Sect. E 64 o256

16. Swamy K C K, Kumaraswamy S and Kommana P 2001 Very Strong $\mathrm{C}-\mathrm{H} \cdots \mathrm{O}, \mathrm{N}-\mathrm{H} \cdots \mathrm{O}$, and $\mathrm{O}-\mathrm{H} \cdots \mathrm{O}$ Hydrogen Bonds Involving a Cyclic Phosphate J. Am. Chem. Soc. 12312642

17. Klussmann M, Ratjen L, Hoffmann S, Wakchaure V, Goddard R and List B 2010 Synthesis of TRIP and Analysis of Phosphate Salt Impurities Synth. Lett. 2189
18. DeFord J, Chu F and Anslin E V 1996 Dimerization constants for phosphoric acid diesters Tetrahedron Lett. 371925

19. Peppard D F, Ferraro J R and Mason G W 1957 Possible hydrogen bonding in certain interactions of organic phosphorus compounds J. Inorg. Nucl. Chem. 4 371

20. Peppard D F, Ferraro J R and Mason G W 1958 Hydrogen bonding in organophosphoric acids J. Inorg. Nucl. Chem. 7231

21. Dar A A, Mallick A and Murugavel R 2015 Synthetic strategies to achieve further-functionalised monoaryl phosphate primary building units: crystal structures and solid-state aggregation behaviour New J. Chem. 39 1186

22. Kalita A C, Sharma K and Murugavel R 2014 Pseudopolymorphism leading and two different supramolecular aggregations in a phosphate monoester: role of a rare water-dimer CrystEngComm 1651

23. Kuczek M, Bryndal I. and Lis T 2006 4-Nitrophenyl phosphoric acid and its four different potassium salts: a solid state structure and kinetic study CrystEngComm $\mathbf{8}$ 150

24. Onoda A, Yamada Y, Okamura T-A, Yamamoto H and Ueyama N 2002 Mononuclear Ca(II)-Bulky ArylPhosphate Monoanion and Dianion Complexes with Ortho-Amide Groups Inorg. Chem. 416038

25. Onoda A, Okamura T, Yamamoto $\mathrm{H}$ and Ueyama $\mathrm{N}$ 2001One-dimensional $\mathrm{P}-\mathrm{OH} \cdots \mathrm{O}=\mathrm{P}$ hydrogen bonds restricted by the bulky molecule 2,6-diiso-propyl-phenyl di-hydrogen phosphate Acta Crystallogr., Sect. E o1022

26. Mehring M, Schurmann M and Ludwig R 2003 tertButylphosphonic Acid: From the Bulk to the Gas Phase Chem. Eur. J. 9837

27. Chandrasekhar V, Sasikumar P, Boomishankar R and Anantharaman G 2006 Assembly of Lipophilic Tetranuclear $\left(\mathrm{Cu}_{4}\right.$ and $\left.\mathrm{Zn}_{4}\right)$ Molecular Metallophosphonates from 2,4,6-Triisopropylphenylphosponic Acid and Pyrazole Ligands Inorg. Chem. 453344

28. Weakley T J R 1976 Benzenephosphonic acid Acta Cryst. B32 2889

29. Parmar D, Sugiono E, Raja S and Rueping M 2014 Complete Field Guide to Asymmetric BINOL-Phosphate Derived Brønsted Acid and Metal Catalysis: History and Classification by Mode of Activation; Brønsted Acidity, Hydrogen Bonding, Ion Pairing, and Metal Phosphates Chem. Rev. 1149047

30. Minyaev M E, Nifantev I E, Tavtorkin A N, Korchagina S A and Zeynalova S S 2015 Crystal structure of [bis(2,6-diisopropylphenyl) phosphato- $\kappa O$ ]tris(methanol-кO)lithium methanol monosolvate Acta Cryst. E71 443

31. Murugavel R, Sathiyendiran M, Pothiraja R, Walawalkar M G, Mallah T and Rivire E 2004 Monomeric, Tetrameric, and Polymeric Copper Di-tert-butyl Phosphate Complexes Containing Pyridine Ancillary Ligands Inorg. Chem. 43945

32. Murugavel R and Shanmugan S 2007 Seeking tetrameric transition metal phosphonate with a D4R core and organising it into a 3-D supramolecular assembly Chem. Commun. 1257 
33. Saxena P, Mandal S K, Sharma K and Murugavel R 2018 Delineating factors that dictate the framework of a bulky phosphate derived metal complexes: Sterics of phosphate, anion of the metal salt and auxiliary N-donor ligand Inorg. Chim. Acta 469353

34. Mandal D, Santra B, Kalita P, Chrysochos N, Malakar A, Narayanan R S, Biswas S, Schulzke C, Chandrasekhar V and Jana A 2017 2,6-(Diphenylmethyl)Aryl-Substituted Neutral and Anionic Phosphates: Approaches to H-Bonded Dimeric Molecular Structures Chem. Select 28898

35. Santra B, Mandal D, Gupta V, Kalita P, Kumar V, Narayanan R S, Dey A, Chrysochos N, Mohammad A, Singh A, Zimmer M, Dalapati R, Biswas S, Schulzke C, Chandrasekhar V, Scheschkewitz D and Jana A 2019 Structural Diversity in Supramolecular Organization of Anionic Phosphate Monoesters: Role of Cations ACS Omega 42118

36. Santra B, Narayanan R S, Kalita P, Kumar V, Mandal D, Gupta V, Zimmer M, Huch V, Chandrasekhar V, Scheschkewitz D, Schulzke C and Jana A 2019 Modulation of the Nuclearity of Molecular Mg(II)Phosphates: Solid-State Structural Change Involving Coordinating Solvents Dalton Trans. 488853
37. Gupta V, Santra B, Mandal D, Das S, Narayanan R S, Kalita P, Rao D K, Schulzke C, Pati S K, Chandrasekhar V and Jana A 2018 Neutral and anionic phosphate-diesters as molecular templates for the encapsulation of a water dimer Chem. Commun. 54 11913

38. Korb M and Lang H 2014 Planar Chirality from the Chiral Pool: Diastereoselective Anionic Phospho-Fries Rearrangements at Ferrocene Organometallics 336643

39. SMART \& SAINT Software Reference manuals, Version 6.45; Bruker Analytical X-ray Systems, Inc.: Madison, WI, 2003

40. Sheldrick G M 1996 SADABS, Program for Empirical Absorption Correction, University of Gottingen, Germany

41. Sheldrick G M 2015 SHELXT - Integrated space-group and crystal structure determination Acta Crystallogr. A71 3

42. Sheldrick G M 2015 Crystal structure refinement with SHELXL Acta Crystallogr. C71 3

43. Dolomanov O V, Bourhis L J, Gildea R J, Howard J A $\mathrm{K}$ and Puschmann H 2009 OLEX2: a complete structure solution, refinement and analysis program J. Appl. Cryst. 42339 